\title{
Quercetin Increases Doxorubicin-Induced Apoptosis through Oxidative DNA Damage in KATO III Gastric Cancer Cells
}

\author{
Mingxiang Chen ${ }^{1, *}$, Chunxiao Duan ${ }^{1}$ and Jun Pan ${ }^{1, *}$ \\ 1 Department of Oncology, Yizheng People's Hospital, Jiangsu Province, China \\ * Corresponding author: Mingxiang Chen, Department of Oncology, Yizheng People's Hospital, Jiangsu Province, China. Email: \\ cmxy8478@163.com; Jun Pan, Department of Oncology, Yizheng People's Hospital, Jiangsu Province, China. Email: panjun1109@126.com
}

Received 2021 January 09; Revised 2021 February 01; Accepted 2021 March 16.

\begin{abstract}
Background: Gastric cancer is the most common gastrointestinal malignancy with an increasing incidence rate worldwide. Finding novel curative and preventive approaches that could target the tumor cells without affecting the normal cells and overcome drug resistance will be tremendously useful.

Objectives: This study aimed to evaluate the effects of quercetin (QUE) in combination with doxorubicin (DOX) on apoptosis and its underlying mechanisms in the KATO III gastric cancer cell line.

Methods: The effects of Que and DOX on cell viability were measured using an MTT assay. Western blot was used for the measurement of $\gamma \mathrm{H} 2 \mathrm{AX}$ protein expression. The expression levels of 8-Hydroxy-2'-deoxyguanosine were evaluated by enzyme-linked immunosorbent assay. The 2, 7-dichlorofuorescin diacetate fluorescence dye was used to detect the formation of reactive oxygen species (ROS). The activities of antioxidant enzymes (superoxide dismutase, catalase, glutathione peroxidase, glutathione reductase, and glutathione Stransferase) were also assessed. For evaluation of apoptosis, the terminal deoxynucleotidyl transferase dUTP nick end labeling assay was used.

Results: Based on the findings, QUE significantly increased the cytotoxic effects of DOX. Besides, QUE considerably increased the expression levels of $\gamma \mathrm{H} 2 \mathrm{AX}$. Upon QUE treatment, ROS levels increased, and antioxidant enzyme expression levels markedly decreased. Moreover, QUE treatment resulted in the potentiation of doxorubicin-induced apoptosis in KATO III cells, compared to the cells treated with either QUE or DOX.

Conclusion: Overall, co-administration of QUE and DOX enhances cytotoxicity, increases ROS levels, induces oxidative DNA damage, and decreases cellular antioxidant defense, and thereby might promise a therapeutic regimen in promoting the clinical efficacy of the treatment of patients with gastric cancer.
\end{abstract}

Keywords: Apoptosis, Doxorubicin, Oxidative DNA damage, Quercetin, ROS

\section{Background}

Gastric cancer is the most prevalent gastrointestinal malignancy, with more than a million new cases estimated annually worldwide (1). Moreover, since it can only be diagnosed at the advanced stages, its mortality rate is high, making it the third leading cause of cancer-associated deaths (2). Strikingly, gastric cancer is two times more prevalent in males than females (3). The standard treatment for gastric cancer includes a combination of multi-drug chemotherapy, to which a large number of patients respond. In contrast, approximately all patients acquire resistance to chemotherapeutic agents which results in median overall survival of less than one year (4). Furthermore, combination chemotherapies are complicated owing to the diversity in pharmacokinetics, bio-distribution, membrane transport mechanisms, and drug clearance systems $(5,6)$.

Doxorubicin (DOX) is the first-line chemotherapeutic agent, mainly administered to gastric cancer patients, which is slightly effective (7). The DOX toxicity in tumor cells primarily occurs via intercalation with double-strand DNA leading to breakage of strands and repression of both replication and transcription processes (8). The severe side effects and intrinsic or acquired DOX-resistance are pivotal obstacles that have led to the failure of treatment with mono-administration of DOX (9). Besides, chemotherapeutic drugs overexpress reactive oxygen species (ROS) in vitro, which intensifies their genotoxic effects. Activation of ROS-scavenging mechanisms led to the decrease of ROS levels and less DNA damage, resulting in enhanced chemoresistance (10). Hence, promotion of curative methods that potentiate DOX impacts, provide dose mitigation, and preserve normal tissues are crucially required to improve the treatment of gastric cancer patients.

Among anticancer agents, flavonoids are the most studied ones. These compounds can interfere with particular malignancy stages, repress cell growth, and induce programmed cell death in tumor cells $(11,12)$. Quercetin (QUE; with the IUPAC name 3,5,7,3',4'Penta hydroxyl flavone; $\mathrm{C}_{15} \mathrm{H}_{10} \mathrm{O}_{7}$ ) is one of the most abundant flavonoids present in many vegetables, seeds, and fruits (13). Conflicting findings have been reported in cell cycle arrest by QUE; for instance, it appears that QUE can cause cell cycle arrest at G0/G1 in leukemia, $\mathrm{S}$ phase in colorectal cancer, or $\mathrm{G} 2 / \mathrm{M}$ phases in breast cancer, and oesophageal 
adenocarcinoma cells $(14,15)$.

Aside from the antioxidant activities of QUE, it has an apoptotic effect on neoplasm cells and can hinder tumor expansion (16). Furthermore, QUE has been used in combination with many chemotherapy agents, including DOX and cisplatin $(17,18)$. Due to its lipophilic nature, QUE can traverse the cell membrane and initiate synergistic effects on DOX and other chemotherapeutic agents (19-21).

\section{Objectives}

The functional mechanism of QUE in combination with DOX during the induction of apoptosis in gastric cancer is mostly unclear. Therefore, this study aimed to scrutinize the effects of QUE in combination with DOX on apoptosis and its underlying mechanisms in KATO III gastric tumor cells.

\section{Methods}

\subsection{Materials and reagents}

KATO III cell line was purchased from Sun Yat-sen University Cancer Center (Guangzhou, China) and fetal bovine serum (FBS) was provided by Thermo Fisher Scientific (Waltham, MA). Moreover, RPMI-1640 media, phosphate-buffered saline (PBS), and TrypsinEDTA were bought from Gibco Life Technologies (Gibco, USA). Antibiotics, dimethyl sulfoxide (DMSO), and trypan blue were purchased from Sigma Aldrich Co., USA. In addition, Bio Basic Co. provided 3-(4,5Dimethylthiazol-2-yl)-2,5-diphenyltetrazolium.

bromide (MTT). Furthermore, 8-oxo-7,8-dihydro-2' deoxyguanosine (8-oxo-dG, ab201734) enzyme-linked immunosorbent assay (ELISA) competitive and genomic DNA isolation were bought from the Abcam Co., Cambridge, UK. Besides, ROS, catalase (CAT), superoxide dismutase (SOD), glutathione (GSH) peroxidase (GPx), GSH s-transferase (GST, E BC K029), and GSH reductase (GR, E-BC-K099) assay kits were purchased from the Elabscience Biotechnology Co. Wuhan, China. Primary monoclonal antibody against $\gamma$ H2AX from Boster Biological Technology (USA, Cat. No. \#M00241) and $\beta$-actin were provided by Santa Cruz Biotech (Cat. No. sc-130300).

\subsection{Cell culture}

The KATO III cells were seeded in RPMI-1640 (+2 mM L-glutamine) accompanied by $10 \%$ FBS, $1 \%$ pen/strep at $37{ }^{\circ} \mathrm{C}$ with $95 \% \mathrm{O}_{2}$, and $5 \% \mathrm{CO}_{2}$ in the incubator. Cells in the logarithmic phase were collected for functional analysis, and the number of surviving cells was defined by performing the trypan blue dye exclusion test.

\subsection{MTT assay}

Cell viability was performed using the MTT assay. The KATO III cells were seeded in a 96-well microplate overnight at the density of $2 \times 10^{4}$ cells $/ 200 \mu \mathrm{l} /$ well their growth capability was evaluated. Afterward, the cells were exposed to a serum-containing medium with QUE $(0-125 \mu \mathrm{M})$ and DOX (0-1.25 $\mu \mathrm{mol} / \mathrm{L})$ alone or in combination for $48 \mathrm{~h}$. At the end of the treatment, cells were exposed to $5 \mathrm{mg} / \mathrm{mL}$ of MTT $(20 \mu \mathrm{l})$ for $4 \mathrm{~h}$. Subsequently, $150 \mu \mathrm{l}$ of DMSO was added for solubilization of formazan crystals, followed by persistent shaking for $20 \mathrm{~min}$ at $37^{\circ} \mathrm{C}$. Afterward, the absorbance value was detected at $570 \mathrm{~nm}$ by ELx808 plate auto-reader.

\subsection{Evaluation of 8-Hydroxy-2'-deoxyguanosine levels}

For measurement of 8-Hydroxy-2'-deoxyguanosine amounts as a marker of DNA damage, we extracted DNA from KATO III cancer cells by commercial DNA extraction kit. An ELISA kit was employed for the detection of this marker.

\subsection{Measurement of reactive oxygen species levels}

Effects of various treatments on intracellular ROS levels were evaluated by ROS Assay kit. For this purpose, cells were incubated with QUE, DOX, and their combination, washed with PBS at $\mathrm{pH} 7.4$, and then incubated with $10 \mu \mathrm{mol} / \mathrm{L}$ of 2,7 dichlorofuorescin diacetate (DCFH-DA) for $40 \mathrm{~min}$ at $37^{\circ} \mathrm{C}$ in the dark. Intracellular ROS was reacted with DCFH-DA which resulted in a highly fluorescent compound dichlorofluorescein (DCF). The DCF fluorescence intensity was assessed with excitation wavelength at $485 \mathrm{~nm}$ and emission wavelength at $525 \mathrm{~nm}$. The data are presented as relative DCF fluorescence (ratio DCF-induced fluorescence/DCFinduced control fluorescence).

\subsection{Evaluation of glutathione peroxidase activity}

The GPx activity was evaluated based on the protocol. In this assay, GPx catalyzes the reaction between $\mathrm{H}_{2} \mathrm{O}_{2}$ and GSH to generate $\mathrm{H}_{2} \mathrm{O}$ and oxidized GSH (GSSG). The rate of enzymatic reaction can be used for the evaluation of the GPx activity. Hence, the activity of GSH can be determined by calculating the consumption of alleviated GSH levels. Since H2O2 and diminished GSH can react without catalysis of GPx, the part of GSH decrement via non-enzymatic reaction should be decreased.

The reaction of GSH with dinitrobenzoic acid can produce 5-thio-dinitrobenzoic acid anion that has a stable golden color. The absorbance value was read at $412 \mathrm{~nm}$ using an ELISA plate reader. Enzyme-specific activities were expressed as units/mg of protein. According to previous research, one unit of GPx in 1 $\mathrm{mg}$ of protein can catalyze the consumption of 1 $\mu \mathrm{mol} / \mathrm{L}$ GSH with deduction of the effect of nonenzyme reaction at $37^{\circ} \mathrm{C}$ for five min.

\subsection{Measurement of glutathione s-transferase activity}

The GST activity was evaluated using the GST assay kit. In this kit, the activity of GST was measured with regard to the fact that GST catalyzes 
the binding of GSH to 1-chlorine-2, 4-dinitrobenzene. It should be noted that the activity of GST is linearly correlated with the change of GSH level. The absorbance value was detected at $412 \mathrm{~nm}$ using a plate auto-reader.

\subsection{Measurement of Glutathione reductase activity}

Glutathione reductase (GR) activity could be detected indirectly by a coupled reaction with GSH. The GSSG is recycled to its diminished state by GR and nicotinamide adenine dinucleotide phosphate (NADPH). Oxidation of NADPH to $\mathrm{NADP}^{+}$is accompanied by a decline in absorbance at $340 \mathrm{~nm}$. Hence, the rate of reduction in absorbance is directly proportional to the GR activity in the sample. Enzyme-specific activities were expressed as units/mg of protein. The amount of enzyme of 1 mmol of NADPH catalyzed by $1 \mathrm{~g}$ protein per min is defined as 1 unit.

\subsection{Western blotting}

After appropriate exposure to drugs, the total cellular proteins were trypsinized and extracted from the cells using radioimmunoprecipitation assay lysis buffer. Afterward, centrifugation 114,000 $\times$ g) at $4{ }^{\circ} \mathrm{C}$ for 20 min was employed for harvesting the lysates and protein concentration was determined using the Bradford method. In total, 50 $\mu \mathrm{g}$ of protein was separated in a $10 \%$ sodium dodecyl sulphate-polyacrylamide gel electrophoresis and electrophoretically transferred to polyvinylidene difluoride (PVDF) membrane. Afterward, the membranes were blocked with $5 \%$ skim milk in Tris-buffered saline, $0.1 \%$ Tween 20 (TBST) for one $\mathrm{h}$ and incubated with primary antibody against $\gamma$ $\mathrm{H} 2 \mathrm{AX}$ overnight at $4{ }^{\circ} \mathrm{C}$.

After being washed with TBST, the membranes were incubated with anti-rabbit or anti-mouse IgGconjugated secondary antibodies for one $h$. Blots were visualized by ImageJ software (version 1.42q) was used for Band analysis.

\subsection{Statistical analysis}

Results are expressed as mean $\pm \mathrm{SD}$, and a p-value of less than 0.05 was considered statistically significant. All quantitative results were processed by GraphPad Prism software (version 7.04). Kolmogorov-Smirnov and Levene tests were used to evaluate the normality of the results. The groups were compared using the one-way ANOVA post-hoc (Tukey and Dunnett) test.

\section{Results}

4.1. Co-administration of quercetin and doxorubicin could significantly decrease cellular proliferation of KATO III cells

Inhibitory effects of QUE and DOX on cell proliferation of KATO III cells were scrutinized by the MTT assay. As presented in Figures $1 \mathrm{~A}$ and $1 \mathrm{~B}$, different concentrations of QUE $(0,25,50,75,100$, and $125 \mu \mathrm{M})$ and DOX $(0,0.25,0.5,0.75,1$, and $1.25 \mu \mathrm{M})$ were used during $48 \mathrm{~h}$. Results revealed the dramatic reduction of cell viability of KATO III cells in a concentrationdependent manner. The IC50 values of QUE and DOX in KATO III cells were $50.37 \mu \mathrm{M}$ and $0.87 \mu \mathrm{M}$, respectively. After combination with Que in a concentrationdependent manner, the IC50 value of DOX was 0.64 which decreased in comparison to DOX alone $(\mathrm{P}<0.05)$. In a sense, $50.37 \mu \mathrm{M}$ of QUE and $0.64 \mu \mathrm{M}$ of DOX killed $50 \%$ of KATO III cell lines after $48 \mathrm{~h}$ (Figure 1C).

4.2. Co-administration of quercetin and doxorubicin promotes intracellular reactive oxygen species levels in KATO III cells in gastric cancer

To elucidate whether ROS generation is responsible for QUE and DOX-induced cell death, we
A

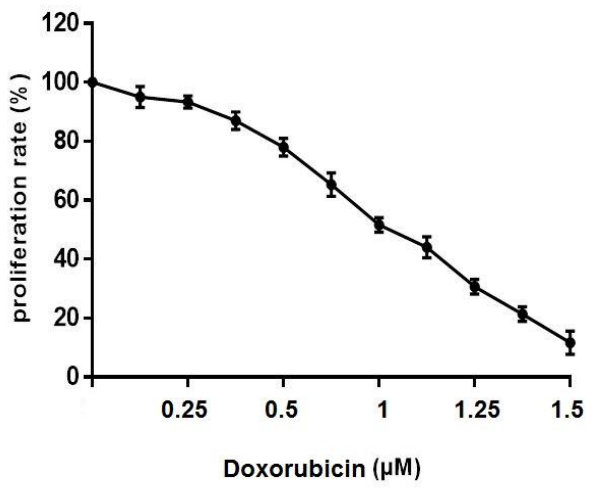

B

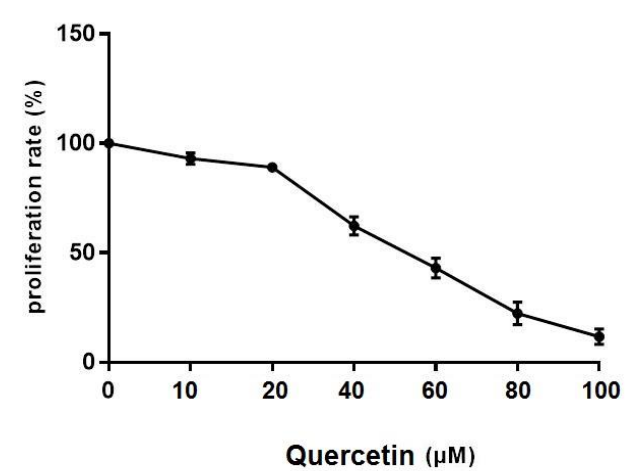

Figure 1. Effects of quercetin and doxorubicin and their combination on cell proliferation. All values are shown as mean \pm SD. 


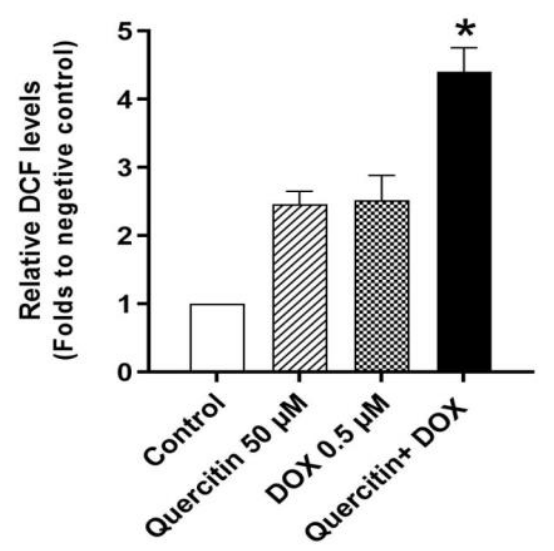

Figure 2. Effects of quercetin and doxorubicin (DOX) and their combination on reactive oxygen species levels. All values are shown as mean \pm SD. DCF: dichlorofluorescein

examined the effect of their mono-administration and co-administration on the ROS generation using fluorescent probe procedure, DCFH-DA. It was found that QUE and DOX potently elevated the ROS levels, compared to the control group. Moreover, the combination of QUE and DOX had a more powerful impact $(\mathrm{P}<0.05$; Figure 2$)$.

\subsection{Co-administration of quercetin and doxorubicin} promotes DNA damage in KATO III cells in gastric cancer

To ascertain the effects of QUE, DOX, and their combination on DNA damage in KATO III cells, we surveyed 8-OXO-dG levels as a valid biomarker of DNA oxidation. Our results showed that QUE and DOX increased the 8-OXO-dG levels $(\mathrm{P}<0.05$; Figure 3A). Notably, QUE and DOX in combination exerted a more potent effect on increasing the DNA damage $(\mathrm{P}<0.05$; Figure $3 \mathrm{~A})$. Furthermore, the expression levels of $\gamma \mathrm{H} 2 \mathrm{AX}$ that played a substantial role in DNA damage response were also evaluated in this study. The findings demonstrated that QUE and DOX monoadministration resulted in a dramatic increment in $\gamma \mathrm{H} 2 \mathrm{AX}$ levels in both groups $(\mathrm{P}<0.05$; Figure 3B and $\mathrm{C}$ ). Additionally, co-administration of QUE and DOX increased $\gamma \mathrm{H} 2 \mathrm{AX}$ levels more drastically $(\mathrm{P}<0.05$; Figure $3 \mathrm{~B}$ and $\mathrm{C})$.

4.4. Co-administration of quercetin and doxorubicin tackled the activities of the antioxidant enzymes in KATO III cells in gastric cancer

We assessed the activity of antioxidant enzymes, such as CAT, SOD, GST, GR, and GPx. As shown in Figure 4, the collected data indicated that the coadministration of QUE and DOX dramatically repressed the above-mentioned enzymatic activities, compared to the mono-administration $(\mathrm{P}<0.05)$.

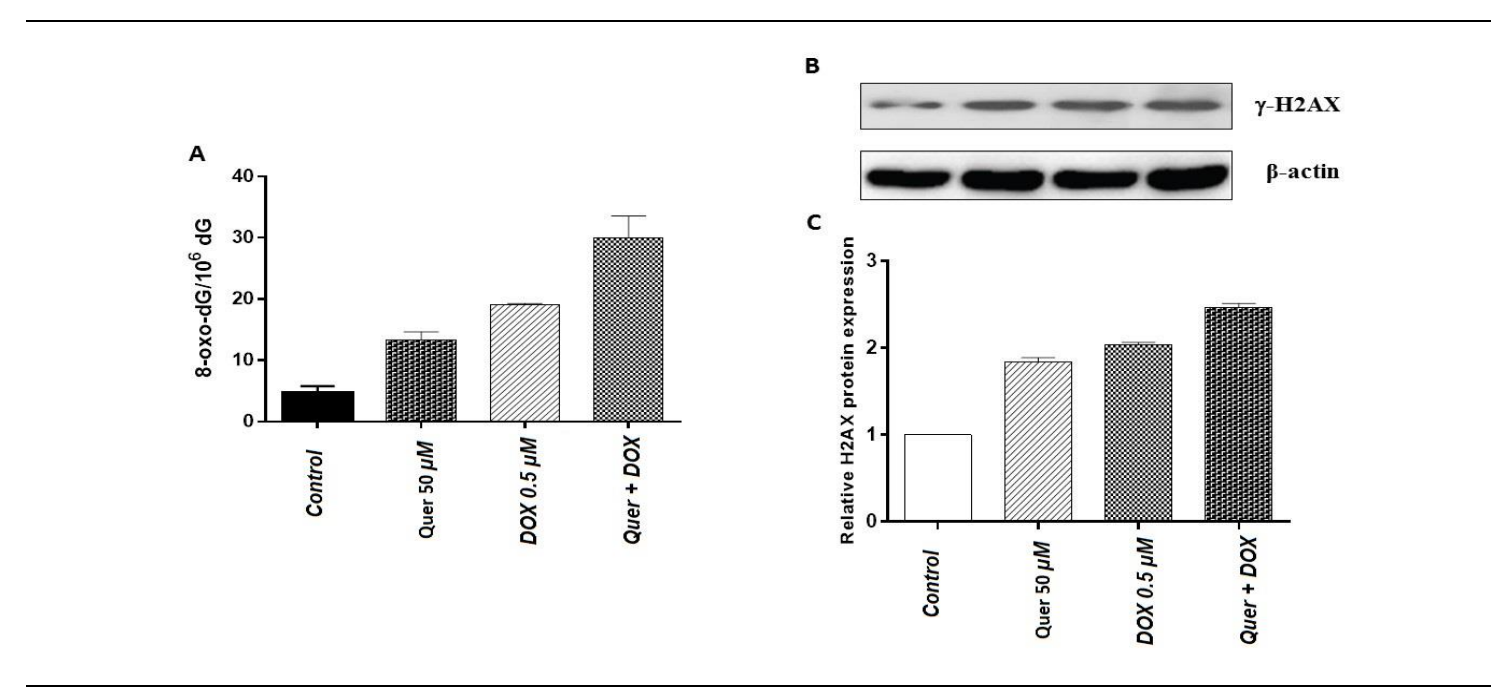

Figure 3. Effects of quercetin (Quer) and doxorubicin (DOX) and their combination on DNA damage. A) Levels of 8-oxo$\mathrm{dG}$ in stomach DNA in the studied groups, B) protein levels of $\gamma$-H2AX. $\beta$-actin was applied as the loading control, C) quantitative interpretation of $\gamma-\mathrm{H} 2 \mathrm{AX}$. All values are shown as mean $\pm \mathrm{SD}$. 


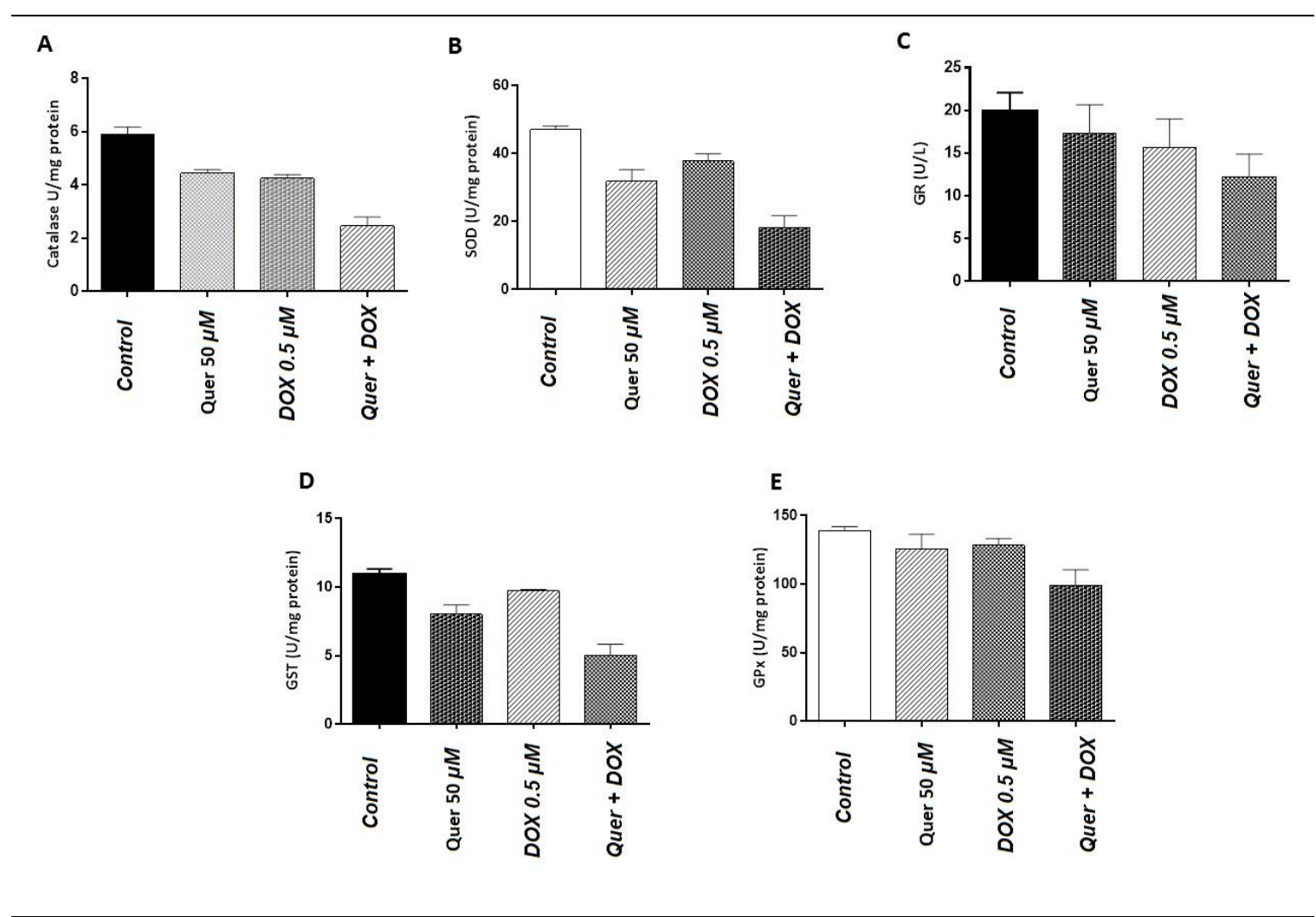

Figure 4. Effects of quercetin (Quer) and doxorubicin (DOX) and their combination on the activity levels of antioxidant enzymes including (A) catalase, (B) SOD, (C) GR, (D) GST, and (E) GPx. Results are shown as mean \pm SD. SOD: superoxide dismutase, GR: glutathione reductase, GPx: glutathione peroxidase, and GST: glutathione S-transferase.

\section{Discussion}

Findings of the present study implied three significant results that allow us to better understand the effects of co-administration of QUE and DOX on the increase in apoptosis through oxidative DNA damage in the KATO III gastric cancer cell line. Firstly, it was found that QUE could significantly diminish the IC50 value of DOX in the KATO III cells, compared to its monoadministration.

Secondly, it was indicated that the coadministration of QUE and DOX had a more potent effect on apoptosis induction. Thirdly, this coadministration dramatically enhanced the 8-0XO$\mathrm{dG}$ and ROS levels and also diminished antioxidant enzyme levels in comparison with monoadministration. These valuable findings highlighted that the co-administration of QUE and DOX exacerbated in vitro apoptosis in the gastric cells.

Although DOX is a potent anticancer agent, its clinical utility is restricted due to its toxicity to healthy tissues. Besides, drug-resistance development is another limitation in the employment of this agent in the eradication of tumor cells (22). Therefore, expansion of the novel, effective, and safe agents with lesser cytotoxicity and side effects are required for tumor treatment (23). It has been well documented that QUE has extensive therapeutic effects, including antiinfective and antioxidant activities. Moreover, apart from its potent effects, QUE induced little or no toxicity in healthy tissues (8).

Based on the results of previous studies, QUE induced toxicity in many human cancer cell lines with differing sensitivities $(15,24,25)$. Furthermore, it has been revealed that QUE affects several cancers by intensifying the DOX activity. Co-administration of QUE and DOX is highly effective against several tumor types, including human lymphoid, myeloid leukemia, and breast cancer cell lines, since it causes repression of cell growth and invasion through the repression of P-glycoprotein $(17,21)$. Herein, the collected data indicated that the combination of QUE and DOX could markedly diminish the IC50 value of DOX in the KATO III cells, compared to the mono-administration of DOX.

The ROS is a pivotal regulator of cellular signal transduction; hence, disturbance of the balance between the producing and cleansing ability of ROS might contribute to cell damage. In a sense, ROS can convert lipids or proteins in the cells into oxidized forms or transform nucleic acids into mutated forms (26). According to previous studies, ROS exert dual function in the cellular process in a concentration-dependent manner since in moderate levels they play a role in cancer onset and development through inflammation, DNA mutation, and cellular damage, while in higher levels they act 
as an anticancer agent by induction of apoptosis (27). Hence, this has led to the conclusion that ROS modulators could be employed as primary cancer impediments to improve treatment plans (10).

The ROS attack guanine in the DNA and transform into 8-0X0-dG that can bind to $\mathrm{T}$ rather than $\mathrm{C}$; hence, the increased level of $8-0 \mathrm{XO}-\mathrm{dG}$ is primarily considered hallmark mutagenesis resulting in oxidative stress (OS) (28). Since 8-OXO$\mathrm{dG}$ is regarded as a suitable biomarker to assess DNA damage in vitro (29), this factor was evaluated in response to co-administration in this study. As mentioned earlier, we found that 8-OXO-dG elevated in line with ROS overproduction.

Based on the results of previous studies, anticancer properties of DOX, at least in part, mediated through the induction of oxidative DNA damage, which is in line with those of the present study. Findings of the present study indicated that QUE, combined with DOX, exerts more substantial DNA damage in KATO III cells, which subsequently brings about potent apoptosis. In another aspect, Ramasamy et al. demonstrated that the coadministration of QUE and DOX using $\mathrm{pH}$-sensitive polypeptide-based nanocarriers enabled the QUE to dramatically enhance the cytotoxic impression of DOX and additionally induce apoptosis in the tumor cells (30).

Following the intercalation of DOX into the DNA, the structural changes impede a convenient interaction of topoisomerase II with DNA, ultimately impairing ataxia telangiectasia mutated activation resulting in $\gamma \mathrm{H} 2 \mathrm{AX}$ formation $(23,31)$. Phosphorylation of histone H2AX on Ser-139 $(\gamma \mathrm{H} 2 \mathrm{AX})$ is a sensitive biomarker of DNA injury and DNA damage response, especially the stimulation of DNA double-strand breaks. In this regard, the Western blot analysis of $\gamma \mathrm{H} 2 \mathrm{AX}$ revealed that exposure of cells to Que or DOX led to an increase in $\gamma \mathrm{H} 2 \mathrm{AX}$ in KATO III cells, which was reflective of the findings of the present and previous studies $(32,33)$. Moreover, co-administration of QUE and DOX results in a significant enhancement of $\gamma \mathrm{H} 2 \mathrm{AX}$ levels.

Importantly, QUE, combined with DOX, leads to remarkable alleviation in antioxidant levels, including SOD, catalase, GPx, GR, and GSH $S$ transferase. Indeed, decreasing intracellular GSH enhances ROS and improves chemotherapy sensitivity. It has been widely accepted that several chemotherapeutic agents and adjuvant therapies cause a decrease in antioxidant capacity and blockage of antioxidant defense, resulting in cell death (34).

Furthermore, our results supported this notion that programmed cell death is the consequence of ROS overproduction and antioxidant defense inhibition, which is considered one of the essential mechanisms in QUE anticancer activity (35).
Furthermore, Staedler et al (21). found that DOX, when combined with QUE in the breast cancer cell lines, synergistically repressed cell growth and induced apoptosis via decrement S-transferase of GR GSH (36). Indeed, over-produced antioxidant enzymes led to the improvement of the tumor cell and prevention of DNA damage, OS, and repression of apoptosis; these conditions can result in resistance to chemotherapies (37).

\section{Conclusion}

Based on the findings, QUE could induce cytotoxicity through various methods. Moreover, recent findings have revealed that QUE, a DNA intercalator, increases the cytotoxicity of doxorubicin-induced apoptosis via oxidative DNA damage in KATO III gastric cancer cells. Consequently, our data imply that QUE is a hopeful candidate for the treatment of gastric cancer with apoptosis induction.

\section{Acknowledgments}

This research was supported by Yizheng People's Hospital, Jiangsu Province, China.

\section{Footnotes}

Author's contributions: M.C performed the experiments and supervision. C.D analyzed the data. J.P designed experiments and co-wrote the paper.

Conflicts of Interest: The authors have no conflict of interest to declare.

Ethical Approval: This study was approved by Yizheng People's Hospital, Jiangsu Province, China.

Funding/Support: Yizheng People's Hospital, Jiangsu Province, China.

Informed Consent: Informed consent was not necessary for this in vitro study.

\section{References}

1. Thrift AP, El-Serag HB. Burden of gastric cancer. Clin Gastroenterol Hepatol. 2020;18(3):534-42. doi: 10.1016/ j.cgh.2019.07.045. [PubMed: 31362118].

2. Lochhead P, El-Omar EM. Molecular predictors of gastric neoplastic progression. Cancer Cell. 2018;33(1):9-11. doi: 10.1016/j.ccell.2017.12.006. [PubMed: 29316435].

3. Smyth EC, Nilsson M, Grabsch HI, van Grieken NC, Lordick F. Gastric cancer. Lancet. 2020;396(10251):635-48. doi: 10.1016/S0140-6736(20)31288-5. [PubMed: 32861308].

4. Lin JX, Yoon C, Li P, Ryeom SW, Cho SJ, Zheng $\mathrm{CH}$, et al CDK5RAP3 as tumour suppressor negatively regulates selfrenewal and invasion and is regulated by ERK1/2 signalling in human gastric cancer. Br J Cancer. 2020;123(7):1131-44. doi: 10.1038/s41416-020-0963-y. [PubMed: 32606358].

5. Zhang L, Lu J, Jin Y, Qiu L. Folate-conjugated beta-cyclodextrinbased polymeric micelles with enhanced doxorubicin antitumor efficacy. Colloids Surf B Biointerfaces. 2014; 122:260-9. doi: 10.1016/j.colsurfb.2014.07.005. [PubMed: 25058857].

6. Wang B, Yu XC, Xu SF, Xu M. Paclitaxel and etoposide co- 
loaded polymeric nanoparticles for the effective combination therapy against human osteosarcoma. J Nanobiotechnology. 2015;13:22. doi: 10.1186/s12951-015-0086-4. [PubMed: 25880868].

7. Qi W, Boliang $\mathrm{W}$, Xiaoxi T, Guoqiang $\mathrm{F}$, Jianbo X, Gang W. Cardamonin protects against doxorubicin-induced cardiotoxicity in mice by restraining oxidative stress and inflammation associated with Nrf2 signaling. Biomed Pharmacother. 2020;122:109547. doi: 10.1016/j.biopha.2019.109547. [PubMed: 31918264].

8. Darband SG, Kaviani M, Yousefi B, Sadighparvar S, Pakdel FG, Attari JA, et al. Quercetin: a functional dietary flavonoid with potential chemo-preventive properties in colorectal cancer. $J$ Cell Physiol. 2018;233(9):6544-60. doi: 10.1002/jcp.26595. [PubMed: 29663361].

9. Hafez HM, Hassanein H. Montelukast ameliorates doxorubicin-induced cardiotoxicity via modulation of $\mathrm{p}$ glycoprotein and inhibition of ROS-mediated TNF- $\alpha / N F-\kappa B$ pathways. Drug Chem Toxicol. 2020;27:1-12. doi: 10.1080/01480545.2020.1730885. [PubMed: 32106718].

10. Srinivas US, Tan BW, Vellayappan BA, Jeyasekharan AD. ROS and the DNA damage response in cancer. Redox Biol. 2019;25:101084. doi: 10.1016/j.redox.2018.101084. [PubMed: 30612957].

11. Dong Q, Chen L, Lu Q, Sharma S, Li L, Morimoto S, et al. Quercetin attenuates doxorubicin cardiotoxicity by modulating B mi-1 expression. Br J Pharmacol. 2014; 171(19):4440-54. doi: 10.1111/bph.12795.

12. Henidi HA, Al-Abbasi FA, El-Moselhy MA, El-Bassossy HM, AlAbd AM. Despite blocking doxorubicin-induced vascular damage, quercetin ameliorates its antibreast cancer activity. Oxid Med Cell Longev. 2020;2020:8157640. doi: 10.1155/2020/8157640. [PubMed: 33728016].

13. Sharma A, Parikh M, Shah H, Gandhi T. Modulation of Nrf2 by quercetin in doxorubicin-treated rats. Heliyon. 2020; 6(4):e03803. doi: 10.1016/j.heliyon.2020.e03803. [PubMed: 32337383].

14. Zhang Q, Zhao XH, Wang ZJ. Flavones and flavonols exert cytotoxic effects on a human oesophageal adenocarcinoma cell line (OE33) by causing G2/M arrest and inducing apoptosis. Food Chem Toxicol. 2008;46(6):2042-53. doi: 10.1016/j.fct.2008.01.049. [PubMed: 18331776].

15. Srivastava S, Somasagara RR, Hegde M, Nishana M, Tadi SK, Srivastava M, et al. Quercetin, a natural flavonoid interacts with DNA, arrests cell cycle and causes tumor regression by activating mitochondrial pathway of apoptosis. Sci Rep. 2016;6:24049. doi: 10.1038/srep24049. [PubMed: 27068577].

16. Singh CK, Chhabra G, Ndiaye MA, Siddiqui IA, Panackal JE, Mintie CA, et al. Quercetin-resveratrol combination for prostate cancer management in TRAMP mice. Cancers. 2020;12(8):2141. doi: 10.3390/cancers12082141. [PubMed: 32748838].

17. Wang G, Zhang J, Liu L, Sharma S, Dong Q. Quercetin potentiates doxorubicin mediated antitumor effects against liver cancer through p53/Bcl-xl. PloS One. 2012;7(12): e51764. doi: 10.1371/journal.pone.0051764. [PubMed: 23240061].

18. Nessa MU, Beale P, Chan C, Yu JQ, Huq F. Synergism from combinations of cisplatin and oxaliplatin with quercetin and thymoquinone in human ovarian tumour models. Anticancer Res. 2011;31(11):3789-97. [PubMed: 22110201].

19. Lan H, Hong W, Fan P, Qian D, Zhu J, Bai B. Quercetin inhibits cell migration and invasion in human osteosarcoma cells. Cell Physiol Biochem. 2017;43(2):553-67. doi: 10.1159/000480528. [PubMed: 28965117].

20. Srivastava S, Somasagara RR, Hegde M, Nishana M, Tadi SK, Srivastava M, et al. Quercetin, a natural flavonoid interacts with DNA, arrests cell cycle and causes tumor regression by activating mitochondrial pathway of apoptosis. Sci Rep. 2016;6:24049. doi: 10.1038/srep24049. [PubMed: 27068577].

21. Staedler D, Idrizi E, Kenzaoui BH, Juillerat-Jeanneret L. Drug combinations with quercetin: doxorubicin plus quercetin in human breast cancer cells. Cancer Chemother Pharmacol.
2011;68(5):1161-72. doi: 10.1007/s00280-011-1596-x. [PubMed: 21400027].

22. Hong HJ, Liu JC, Chen PY, Chen JJ, Chan P, Cheng TH. Tanshinone IIA prevents doxorubicin-induced cardiomyocyte apoptosis through Akt-dependent pathway. Int I Cardiol. 2012; 157(2):174-9. doi: 10.1016/j.ijcard.2010.12.012. [PubMed: 21190747].

23. Majidinia M, Bishayee A, Yousefi B. Polyphenols: major regulators of key components of DNA damage response in cancer. DNA Repair. 2019;82:102679. doi: 10.1016/j.dnarep. 2019.102679. [PubMed: 31450085].

24. Choi JA, Kim JY, Lee JY, Kang CM, Kwon HJ, Yoo YD, et al Induction of cell cycle arrest and apoptosis in human breast cancer cells by quercetin. Int J Oncol. 2001;19(4):837-44. doi: 10.3892/ijo.19.4.837. [PubMed: 11562764].

25. Mertens-Talcott SU, Percival SS. Ellagic acid and quercetin interact synergistically with resveratrol in the induction of apoptosis and cause transient cell cycle arrest in human leukemia cells. Cancer Lett. 2005;218(2):141-51. doi: 10.1016/j.canlet.2004.06.007. [PubMed: 15670891].

26. Ock CY, Kim EH, Choi DJ, Lee HJ, Hahm KB, Chung MH. 8Hydroxydeoxyguanosine: not mere biomarker for oxidative stress, but remedy for oxidative stress-implicated gastrointestinal diseases. World J Gastroenterol. 2012;18(4):302-8. doi: 10.3748/wjg.v18.i4.302. [PubMed: 22294836].

27. Lin S, Li Y, Zamyatnin Jr AA, Werner J, Bazhin AV. Reactive oxygen species and colorectal cancer. J Cell Physiol 2018;233(7):5119-32. doi: 10.1002/jcp.26356. [PubMed: 29215746].

28. Huang T, Zhou F, Yuan X, Yang T, Liang X, Wang Y, et al. Reactive oxygen species are involved in the development of gastric cancer and gastric cancer-related depression through abl1-mediated inflammation signaling pathway. Oxid Med Cell Longev. 2019;2019:5813985. doi: 10.1155/2019/5813985. [PubMed: 31396300].

29. Guo C, Li X, Wang R, Yu J, Ye M, Mao L, et al. Association between oxidative DNA damage and risk of colorectal cancer: sensitive determination of urinary 8-hydroxy-2'deoxyguanosine by UPLC-MS/MS analysis. Sci Rep. 2016; 6:32581. doi: 10.1038/srep32581. [PubMed: 27585556].

30. Ramasamy T, Ruttala HB, Chitrapriya N, Poudal BK, Choi JY, Kim ST, et al. Engineering of cell microenvironmentresponsive polypeptide nanovehicle co-encapsulating a synergistic combination of small molecules for effective chemotherapy in solid tumors. Acta Biomater. 2017;48:13143. doi: 10.1016/j.actbio.2016.10.034. [PubMed: 27794477].

31. Huelsenbeck SC, Schorr A, Roos WP, Huelsenbeck J, Henninger C, Kaina B, et al. Rac1 protein signaling is required for DNA damage response stimulated by topoisomerase II poisons. J Biol Chem. 2012;287(46):385909. doi: 10.1074/jbc.M112.377903. [PubMed: 23012366]

32. Huang $X$, Kurose $A$, Tanaka $T$, Traganos F, Dai $W$ Darzynkiewicz Z. Activation of ATM and histone H2AX phosphorylation induced by mitoxantrone but not by topotecan is prevented by the antioxidant N-acetyl-Lcysteine. Cancer Biol Ther. 2006;5(8):959-64. doi: 10.4161/cbt.5.8.2878. [PubMed: 16760673].

33. Ikeda M, Kurose A, Takatori E, Sugiyama T, Traganos F, Darzynkiewicz Z, et al. DNA damage detected with $\gamma \mathrm{H} 2 \mathrm{AX}$ in endometrioid adenocarcinoma cell lines. Int J Oncol. 2010;36(5):1081-8. doi: 10.3892/ijo_00000589. [PubMed: 20372780].

34. Sznarkowska A, Kostecka A, Meller K, Bielawski KP. Inhibition of cancer antioxidant defense by natural compounds. Oncotarget. 2017;8(9):15996-6016. doi: 10.18632/oncotarget.13723. [PubMed: 27911871].

35. Di Bella G, Mascia F, Gualano L, Di Bella L. Melatonin anticancer effects. Int J Mol Sci. 2013;14(2):2410-30. doi: 10.3390/ijms14022410. [PubMed: 23348932].

36. Kuhar M, Imran S, Singh N. Curcumin and quercetin combined with cisplatin to induce apoptosis in human laryngeal carcinoma Hep-2 cells through the mitochondrial pathway. J Cancer Mol. 2007;3(4):121-8. doi: 10.29685/ JCM.200708.0004. 
37. Luceri C, Caderni G, Sanna A, Dolara P. Red wine and black tea polyphenols modulate the expression of cycloxygenase-2, inducible nitric oxide synthase and glutathione-related enzymes in azoxymethane-induced f344 rat colon tumors. J Nutr. 2002;132(6):1376-9. doi: 10.1093/jn/132.6.1376. [PubMed: 12042461]. 